\title{
Shear dynamo problem: Quasilinear kinematic theory
}

\author{
S. Sridhar \\ Raman Research Institute, Sadashivanagar, Bangalore 560 080, Indid \\ Kandaswamy Subramanian \\ IUCAA, Post bag 4, Ganeshkhind, Pune 411 007, Indid
}

(Dated: October 25, 2018)

\begin{abstract}
Large-scale dynamo action due to turbulence in the presence of a linear shear flow is studied. Our treatment is quasilinear and kinematic but is non perturbative in the shear strength. We derive the integro-differential equation for the evolution of the mean magnetic field, by systematic use of the shearing coordinate transformation and the Galilean invariance of the linear shear flow. For non helical turbulence the time evolution of the cross-shear components of the mean field do not depend on any other components excepting themselves. This is valid for any Galilean-invariant velocity field, independent of its dynamics. Hence the shear-current assisted dynamo is essentially absent, although large-scale non helical dynamo action is not ruled out.

PACS numbers: 47.27.W-, 47.65.Md, 52.30.Cv, 95.30.Qd
\end{abstract}

Shear flows and turbulence are ubiquitous in astrophysical systems. Recent work suggests that the presence of shear may open new pathways to the operation of large-scale dynamos $[1,2,3,4,5]$. We present a theory of dynamo action in a shear flow of an incompressible fluid which has random velocity fluctuations due either to freely decaying turbulence or generated through external forcing. Of particular interest is the case of non helical large-scale dynamo action in shear flows. Several direct simulations show that large-scale fields can grow from small seed fields under the combined action of non helical turbulence and background shear flow [1, 2]. However, the interpretation of how such a dynamo works is not yet clear. One possibility that has attracted much attention is the shear-current effect [4], in which extra components of the mean electromotive force (EMF) arise due to shear, which couple components of the mean magnetic field parallel and perpendicular to the shear flow. However there is no convergence yet on whether the sign of the relevant coupling term is such as to obtain a dynamo; some analytic calculations [6, 7] and numerical experiments [1] find that the sign of the shearcurrent term is unfavorable for dynamo action. Moreover, analytic calculations treat shear as a small perturbation. We are interested here in studying the shear dynamo without such a restriction.

Our theory is 'local' in character: In the lab frame we consider a background shear flow whose velocity is unidirectional (along the $X_{2}$ axis) and varies linearly in an orthogonal direction (the $X_{1}$ axis). The linear shear flow has a basic symmetry relating to measurements made by a special subset of all observers, who may be called comoving observers. This symmetry is the invariance of the equations with respect to a group of transformations that is a subgroup of the full Galilean group. It may be referred to as 'shear-restricted Galilean invariance', or Galilean invariance (GI). We introduce and explore the consequences of GI velocity fluctuations; not only are these compatible with the underlying symmetry of the problem, but they are expected to arise naturally. This has profound consequences for dynamo action, because the trans- port coefficients that define the mean EMF become spatially homogeneous in spite of the shear flow. Systematic use of the shearing transformation allows us to develop a theory that is non perturbative in the strength of the background shear. However, we ignore the complications associated with nonlinear interactions, hence MHD turbulence and the small-scale dynamo; so our theory is quasilinear in nature, equivalent to the 'first order smoothing approximation' (FOSA).

Let $\left(\boldsymbol{e}_{1}, \boldsymbol{e}_{2}, \boldsymbol{e}_{3}\right)$ be the unit vectors of a Cartesian coordinate system in the lab frame, $\boldsymbol{X}=\left(X_{1}, X_{2}, X_{3}\right)$ the position vector, and $\tau$ the time. The fluid velocity is given by $\left(-2 A X_{1} \boldsymbol{e}_{2}+\boldsymbol{v}\right)$, where $A$ is the shear parameter and $\boldsymbol{v}(\boldsymbol{X}, \tau)$ is a randomly fluctuating velocity field which is incompressible $(\boldsymbol{\nabla} \cdot \boldsymbol{v}=0)$ and has zero mean $(\langle\boldsymbol{v}\rangle=\mathbf{0})$. The magnetic field has a large-scale (mean field) component $\boldsymbol{B}(\boldsymbol{X}, \tau)$, and a fluctuating field, $\boldsymbol{b}$, with zero mean $(\langle\boldsymbol{b}\rangle=\mathbf{0})$. The evolution of the mean field is governed by

$$
\left(\frac{\partial}{\partial \tau}-2 A X_{1} \frac{\partial}{\partial X_{2}}\right) \boldsymbol{B}+2 A B_{1} \boldsymbol{e}_{2}=\boldsymbol{\nabla} \times \mathcal{E}+\eta \boldsymbol{\nabla}^{2} \boldsymbol{B}
$$

where $\mathcal{E}=\langle\boldsymbol{v} \times \boldsymbol{b}\rangle$ is the mean EMF. Our goal is to calculate $\mathcal{E}$ in terms of the statistical properties of the fluctuating velocity field, which we will do using quasilinear theory. This means solving the equation for $\boldsymbol{b}$ by dropping terms that are quadratic in the fluctuations. We also drop the resistive term, assuming that the correlation times are small compared to the resistive timescale. So our theory is applicable when FOSA is valid [8]. Then $\boldsymbol{b}$ obeys

$$
\left(\frac{\partial}{\partial \tau}-2 A X_{1} \frac{\partial}{\partial X_{2}}\right) \boldsymbol{b}+2 A b_{1} \boldsymbol{e}_{2}=\boldsymbol{\nabla} \times(\boldsymbol{v} \times \boldsymbol{B})
$$

It proves convenient to exchange spatial inhomogeneity for temporal inhomogeneity, so we get rid of the $\left(X_{1} \partial / \partial X_{2}\right)$ term through a shearing transformation to new spacetime variables,

$$
x_{1}=X_{1}, \quad x_{2}=X_{2}+2 A \tau X_{1}, \quad x_{3}=X_{3}, \quad t=\tau
$$


We also define new variables, $\boldsymbol{H}(\boldsymbol{x}, t)=\boldsymbol{B}(\boldsymbol{X}, \tau), \boldsymbol{h}(\boldsymbol{x}, t)=$ $\boldsymbol{b}(\boldsymbol{X}, \tau)$ and $\boldsymbol{u}(\boldsymbol{x}, t)=\boldsymbol{v}(\boldsymbol{X}, \tau)$, which are component-wise equal to the old variables.

Then equation (2) becomes

$$
\begin{aligned}
\frac{\partial \boldsymbol{h}}{\partial t}+2 A h_{1} \boldsymbol{e}_{2}= & \left(\boldsymbol{H} \cdot \frac{\partial}{\partial \boldsymbol{x}}+2 A t H_{1} \frac{\partial}{\partial x_{2}}\right) \boldsymbol{u}- \\
& -\left(\boldsymbol{u} \cdot \frac{\partial}{\partial \boldsymbol{x}}+2 A t u_{1} \frac{\partial}{\partial x_{2}}\right) \boldsymbol{H}
\end{aligned}
$$

Not only do sheared coordinates get rid of spatial inhomogeneity, but in quasilinear theory the evolution equation (4) does not contain spatial derivatives of $\boldsymbol{h}(\boldsymbol{x}, t)$. The equations for $h_{1}$ and $h_{3}$ can be integrated directly. The $h_{1}$ so obtained can be substituted in the equation for $h_{2}$ : there occur doubletime integrals which can be manipulated to give expressions with only single-time integrals, by interchanging the order of the integrals. Then the particular solution for $\boldsymbol{h}(\boldsymbol{x}, t)$ is given in component form by

$$
\begin{aligned}
h_{m} & =\int_{0}^{t} d t^{\prime}\left[u_{m l}^{\prime}-2 A\left(t-t^{\prime}\right) \delta_{m 2} u_{1 l}^{\prime}\right]\left[H_{l}^{\prime}+2 A t^{\prime} \delta_{l 2} H_{1}^{\prime}\right] \\
& -\int_{0}^{t} d t^{\prime}\left[u_{l}^{\prime}+2 A t^{\prime} \delta_{l 2} u_{1}^{\prime}\right]\left[H_{m l}^{\prime}-2 A\left(t-t^{\prime}\right) \delta_{m 2} H_{1 l}^{\prime}\right]
\end{aligned}
$$

where primes denote evaluation at spacetime point $\left(\boldsymbol{x}, t^{\prime}\right)$. We have also used notation $u_{m l}=\left(\partial u_{m} / \partial x_{l}\right)$ and $H_{m l}=$ $\left(\partial H_{m} / \partial x_{l}\right)$.

The expression in equation (5) for $\boldsymbol{h}$ should be substituted in $\mathcal{E}=\langle\boldsymbol{v} \times \boldsymbol{b}\rangle=\langle\boldsymbol{u} \times \boldsymbol{h}\rangle$. Following standard procedure, we allow \langle\rangle to act only on the velocity variables but not the mean field; symbolically, it is assumed that $\langle\boldsymbol{u} \boldsymbol{u} \boldsymbol{H}\rangle=\langle\boldsymbol{u} \boldsymbol{u}\rangle \boldsymbol{H}$. After interchanging the dummy indices $(l, m)$ in the last term, we find that the mean EMF is

$$
\begin{aligned}
& \mathcal{E}_{i}=\int_{0}^{t} d t^{\prime}\left[\widehat{\alpha}_{i l}-2 A\left(t-t^{\prime}\right) \widehat{\beta}_{i l}\right]\left[H_{l}^{\prime}+2 A t^{\prime} \delta_{l 2} H_{1}^{\prime}\right]- \\
& \int_{0}^{t} d t^{\prime}\left[\widehat{\eta}_{i m l}+2 A t^{\prime} \delta_{m 2} \widehat{\eta}_{i 1 l}\right]\left[H_{l m}^{\prime}-2 A\left(t-t^{\prime}\right) \delta_{l 2} H_{1 m}^{\prime}\right]
\end{aligned}
$$

where the transport coefficients $(\widehat{\alpha}, \widehat{\beta}, \widehat{\eta})$ are defined in terms of the $\boldsymbol{u} u$ velocity correlators by

$$
\begin{aligned}
\widehat{\alpha}_{i l}\left(\boldsymbol{x}, t, t^{\prime}\right) & =\epsilon_{i j m}\left\langle u_{j}(\boldsymbol{x}, t) u_{m l}\left(\boldsymbol{x}, t^{\prime}\right)\right\rangle \\
\widehat{\beta}_{i l}\left(\boldsymbol{x}, t, t^{\prime}\right) & =\epsilon_{i j 2}\left\langle u_{j}(\boldsymbol{x}, t) u_{1 l}\left(\boldsymbol{x}, t^{\prime}\right)\right\rangle \\
\widehat{\eta}_{i m l}\left(\boldsymbol{x}, t, t^{\prime}\right) & =\epsilon_{i j l}\left\langle u_{j}(\boldsymbol{x}, t) u_{m}\left(\boldsymbol{x}, t^{\prime}\right)\right\rangle
\end{aligned}
$$

It is physically more transparent to consider velocity statistics in terms of the $\boldsymbol{v} \boldsymbol{v}$ velocity correlators, because this is referred to the lab frame, instead of the sheared coordinates. By definition,

$$
u_{m}(\boldsymbol{x}, t)=v_{m}(\boldsymbol{X}(\boldsymbol{x}, t), t)
$$

where $\boldsymbol{X}(\boldsymbol{x}, t)=\left(x_{1}, x_{2}-2 A t x_{1}, x_{3}\right)$ is the inverse of the shearing transformation given in equation (3). The velocity gradient $u_{m l}$ is

$$
u_{m l}=\left(\frac{\partial}{\partial X_{l}}-2 A \tau \delta_{l 1} \frac{\partial}{\partial X_{2}}\right) v_{m}=v_{m l}-2 A \tau \delta_{l 1} v_{m 2}
$$

where $v_{m l}=\left(\partial v_{m} / \partial X_{l}\right)$. Using equations (8) and (9) in (7),

$$
\begin{aligned}
\widehat{\alpha}_{i l}\left(\boldsymbol{x}, t, t^{\prime}\right)= & \epsilon_{i j m}\left[\left\langle v_{j}(\boldsymbol{X}, t) v_{m l}\left(\boldsymbol{X}^{\prime}, t^{\prime}\right)\right\rangle\right. \\
& \left.-2 A t^{\prime} \delta_{l 1}\left\langle v_{j}(\boldsymbol{X}, t) v_{m 2}\left(\boldsymbol{X}^{\prime}, t^{\prime}\right)\right\rangle\right] \\
\widehat{\beta}_{i l}\left(\boldsymbol{x}, t, t^{\prime}\right)= & \epsilon_{i j 2}\left[\left\langle v_{j}(\boldsymbol{X}, t) v_{1 l}\left(\boldsymbol{X}^{\prime}, t^{\prime}\right)\right\rangle\right. \\
& \left.-2 A t^{\prime} \delta_{l 1}\left\langle v_{j}(\boldsymbol{X}, t) v_{12}\left(\boldsymbol{X}^{\prime}, t^{\prime}\right)\right\rangle\right] \\
\widehat{\eta}_{i m l}\left(\boldsymbol{x}, t, t^{\prime}\right)= & \epsilon_{i j l}\left\langle v_{j}(\boldsymbol{X}, t) v_{m}\left(\boldsymbol{X}^{\prime}, t^{\prime}\right)\right\rangle
\end{aligned}
$$

where the quantities $\boldsymbol{X}=\left(x_{1}, x_{2}-2 A t x_{1}, x_{3}\right)$ and $\boldsymbol{X}^{\prime}=$ $\left(x_{1}, x_{2}-2 A t^{\prime} x_{1}, x_{3}\right)$.

We can arrive at some general conclusions for deltacorrelated-in-time velocity fields. Let the the two-point correlator taken between spacetime points $(\boldsymbol{R}, \tau)$ and $\left(\boldsymbol{R}^{\prime}, \tau^{\prime}\right)$ be $\left\langle v_{i}(\boldsymbol{R}, \tau) v_{j}\left(\boldsymbol{R}^{\prime}, \tau^{\prime}\right)\right\rangle=\delta\left(\tau-\tau^{\prime}\right) T_{i j}\left(\boldsymbol{R}, \boldsymbol{R}^{\prime}, \tau\right)$. We define $T_{i j l}(\boldsymbol{R}, \tau)=\left(\partial T_{i j} / \partial R_{l}^{\prime}\right)_{\boldsymbol{R}^{\prime}=\boldsymbol{R}}$. The delta-function ensures that $\boldsymbol{X}$ and $\boldsymbol{X}^{\prime}$ occuring in the velocity correlators of equation (10) are equal to each other. So $\left\langle v_{i}(\boldsymbol{X}, t) v_{j}\left(\boldsymbol{X}^{\prime}, t^{\prime}\right)\right\rangle=$ $\delta\left(t-t^{\prime}\right) T_{i j}(\boldsymbol{X}, \boldsymbol{X}, t)$, and $\left\langle v_{i}(\boldsymbol{X}, t) v_{j l}\left(\boldsymbol{X}^{\prime}, t^{\prime}\right)\right\rangle=\delta(t-$ $\left.t^{\prime}\right) T_{i j l}(\boldsymbol{X}, t)$. The integrals over time in equation (6) can all be performed, so the mean EMF is

$$
\begin{aligned}
\mathcal{E}_{i} & =\epsilon_{i j m}\left[T_{j m l}-2 A t \delta_{l 1} T_{j m 2}\right]\left[H_{l}+2 A t \delta_{l 2} H_{1}\right] \\
& -\epsilon_{i j l}\left[T_{j m}+2 A t \delta_{m 2} T_{j 1}\right] H_{l m}
\end{aligned}
$$

It is useful to write the EMF in terms of the original variables and lab frame coordinates. To this end we transform

$H_{l m}=\left(\frac{\partial}{\partial X_{m}}-2 A \tau \delta_{m 1} \frac{\partial}{\partial X_{2}}\right) B_{l}=B_{l m}-2 A \tau \delta_{m 1} B_{l 2}$

where $B_{l m}=\left(\partial B_{l} / \partial X_{m}\right)$. Then the explicit dependence of $\mathcal{E}_{i}$ on the shear parameter $A$ cancels out, and mean EMF assumes the simple form,

$$
\mathcal{E}_{i}=\epsilon_{i j m} T_{j m l} B_{l}-\epsilon_{i j l} T_{j m} B_{l m}
$$

which is the familiar expression obtained in the absence of shear. Thus, shear needs time to manifest and, to see the effects of shear explicitly, it is necessary to consider non zero correlation times. Henceforth we consider velocity statistics with finite correlation times.

The linear shear flow has a basic symmetry relating to measurements made by a special subset of all observers. We define a comoving observer as one whose velocity with respect to the lab frame is equal to the velocity of the background shear flow, and whose Cartesian coordinate axes are aligned with those of the lab frame. A comoving observer can be labelled by the 
coordinates, $\boldsymbol{\xi}=\left(\xi_{1}, \xi_{2}, \xi_{3}\right)$, of her origin at time $\tau=0$. Different labels identify different comoving observers and vice versa. As the labels run over all possible values, they exhaust the set of all comoving observers. The origin of the coordinate axes of a comoving observer translates with uniform velocity; its position with respect to the origin of the lab frame is given by

$$
\boldsymbol{X}_{c}(\tau)=\left(\xi_{1}, \xi_{2}-2 A \tau \xi_{1}, \xi_{3}\right)
$$

An event with spacetime coordinates $(\boldsymbol{X}, \tau)$ in the lab frame has spacetime coordinates $(\tilde{\boldsymbol{X}}, \tilde{\tau})$ with respect to the comoving observer, given by

$$
\tilde{\boldsymbol{X}}=\boldsymbol{X}-\boldsymbol{X}_{c}(\tau), \quad \tilde{\tau}=\tau-\tau_{0}
$$

where the arbitrary constant $\tau_{0}$ allows for translation in time as well.

Let $[\tilde{\boldsymbol{B}}(\tilde{\boldsymbol{X}}, \tilde{\tau}), \tilde{\boldsymbol{b}}(\tilde{\boldsymbol{X}}, \tilde{\tau}), \tilde{\boldsymbol{v}}(\tilde{\boldsymbol{X}}, \tilde{\tau})]$ denote the mean, the fluctuating magnetic fields and the fluctuating velocity field, respectively, as measured by the comoving observer. They are all equal to the respective quantities measured in the lab frame: $[\tilde{\boldsymbol{B}}(\tilde{\boldsymbol{X}}, \tilde{\tau}), \tilde{\boldsymbol{b}}(\tilde{\boldsymbol{X}}, \tilde{\tau}), \tilde{\boldsymbol{v}}(\tilde{\boldsymbol{X}}, \tilde{\tau})]=$ $[\boldsymbol{B}(\boldsymbol{X}, \tau), \boldsymbol{b}(\boldsymbol{X}, \tau), \boldsymbol{v}(\boldsymbol{X}, \tau)]$. That this must be true may be understood as follows. Magnetic fields are invariant under non-relativistic boosts, so the mean and fluctuating magnetic fields must be the same in both frames. To see that the fluctuating velocity fields must also be the same in both frames, we note that the total fluid velocity measured by the comoving observer is, by definition, equal to $\left(-2 A \tilde{X} \boldsymbol{e}_{2}+\tilde{\boldsymbol{v}}(\tilde{\boldsymbol{X}}, \tilde{\tau})\right)$. This must be equal to the difference between the velocity in the lab frame, $\left(-2 A X \boldsymbol{e}_{2}+\boldsymbol{v}(\boldsymbol{X}, \tau)\right)$, and $\left(-2 A \xi_{1} \boldsymbol{e}_{2}\right)$, which is the velocity of the comoving observer with respect to the lab frame. Using $\tilde{X}=X-\xi_{1}$, we see that $\tilde{\boldsymbol{v}}(\tilde{\boldsymbol{X}}, \tilde{\tau})=\boldsymbol{v}(\boldsymbol{X}, \tau)$. Equations (1) and (2) are invariant under the simultaneous transformations of spacetime coordinates and fields discussed above. We note that this symmetry property is actually invariance under a subset of the full tenparameter Galilean group, parametrized by the five quantities $\left(\xi_{1}, \xi_{2}, \xi_{3}, \tau_{0}, A\right)$; for brevity we refer to this restricted symmetry as Galilean invariance, or simply GI. There is a fundamental difference between the coordinate transformations associated with GI (equation 15) and the shearing transformation (equation 3). The former relates different comoving observers, whereas the latter describes a time-dependent distortion of the coordinate axes of one observer. Moreover, the relationship between old and new variables is homogeneous for the Galilean transformation, whereas it is inhomogeneous for the shearing transformation.

Naturally occuring processes lead to G-invariant velocity statistics. Let the observer in the lab frame correlate $v_{i}$ at spacetime location $(\boldsymbol{R}, \tau)$ with $v_{j}$ at location $\left(\boldsymbol{R}^{\prime}, \tau^{\prime}\right)$. Now consider a comoving observer, the position vector of whose origin is given by $\boldsymbol{X}_{c}(\tau)$ of equation (14). An identical experiment performed by this observer must yield the same results, the measurements now made at the spacetime points denoted by $\left(\boldsymbol{R}+\boldsymbol{X}_{c}(\tau), \tau\right)$ and $\left(\boldsymbol{R}^{\prime}+\boldsymbol{X}_{c}\left(\tau^{\prime}\right), \tau^{\prime}\right)$ in the lab frame variables. Therefore, a GI two-point velocity correlator must satisfy the condition,

$$
\begin{aligned}
& \left\langle v_{i}(\boldsymbol{R}, \tau) v_{j}\left(\boldsymbol{R}^{\prime}, \tau^{\prime}\right)\right\rangle= \\
& \quad\left\langle v_{i}\left(\boldsymbol{R}+\boldsymbol{X}_{c}(\tau), \tau\right) v_{j}\left(\boldsymbol{R}^{\prime}+\boldsymbol{X}_{c}\left(\tau^{\prime}\right), \tau^{\prime}\right)\right\rangle
\end{aligned}
$$

for all $\left(\boldsymbol{R}, \boldsymbol{R}^{\prime}, \tau, \tau^{\prime}, \boldsymbol{\xi}\right)$. We also have

$$
\begin{aligned}
& \left\langle v_{i}(\boldsymbol{R}, \tau) v_{j l}\left(\boldsymbol{R}^{\prime}, \tau^{\prime}\right)\right\rangle= \\
& \quad\left\langle v_{i}\left(\boldsymbol{R}+\boldsymbol{X}_{c}(\tau), \tau\right) v_{j l}\left(\boldsymbol{R}^{\prime}+\boldsymbol{X}_{c}\left(\tau^{\prime}\right), \tau^{\prime}\right)\right\rangle
\end{aligned}
$$

If we now set $\boldsymbol{R}=\boldsymbol{R}^{\prime}=\mathbf{0}, \tau=t, \tau^{\prime}=t^{\prime}$ and $\boldsymbol{\xi}=\boldsymbol{x}$, we will have $\boldsymbol{X}_{c}(\tau)=\left(x_{1}, x_{2}-2 A t x_{1}, x_{3}\right)$ and $\boldsymbol{X}_{c}\left(\tau^{\prime}\right)=$ $\left(x_{1}, x_{2}-2 A t^{\prime} x_{1}, x_{3}\right)$. Therefore $\boldsymbol{X}_{c}(\tau)$ and $\boldsymbol{X}_{c}\left(\tau^{\prime}\right)$ are equal to $\boldsymbol{X}$ and $\boldsymbol{X}^{\prime}$, which are the quantities that enter as arguments in the velocity correlators of equations (10) defining the transport coefficients. Hence, (reading equations (16) and (17) from right to left), we see that

$$
\begin{aligned}
\left\langle v_{i}(\boldsymbol{X}, t) v_{j}\left(\boldsymbol{X}^{\prime}, t^{\prime}\right)\right\rangle & =\left\langle v_{i}(\mathbf{0}, t) v_{j}\left(\mathbf{0}, t^{\prime}\right)\right\rangle=R_{i j}\left(t, t^{\prime}\right) \\
\left\langle v_{i}(\boldsymbol{X}, t) v_{j l}\left(\boldsymbol{X}^{\prime}, t^{\prime}\right)\right\rangle & =\left\langle v_{i}(\mathbf{0}, t) v_{j l}\left(\mathbf{0}, t^{\prime}\right)\right\rangle=S_{i j l}\left(t, t^{\prime}\right)
\end{aligned}
$$

are independent of space, and are given by the functions $R_{i j}\left(t, t^{\prime}\right)$ and $S_{i j l}\left(t, t^{\prime}\right)$. Symmetry and incompressiblity imply that $R_{i j}\left(t, t^{\prime}\right)=R_{j i}\left(t^{\prime}, t\right)$ and $S_{i j j}\left(t, t^{\prime}\right)=0$. Using equations (18) in equations (10), we find that the GI transport coefficients

$$
\begin{aligned}
\widehat{\alpha}_{i l}\left(t, t^{\prime}\right) & =\epsilon_{i j m}\left[S_{j m l}\left(t, t^{\prime}\right)-2 A t^{\prime} \delta_{l 1} S_{j m 2}\left(t, t^{\prime}\right)\right] \\
\widehat{\beta}_{i l}\left(t, t^{\prime}\right) & =\epsilon_{i j 2}\left[S_{j 1 l}\left(t, t^{\prime}\right)-2 A t^{\prime} \delta_{l 1} S_{j 12}\left(t, t^{\prime}\right)\right] \\
\widehat{\eta}_{i m l}\left(t, t^{\prime}\right) & =\epsilon_{i j l} R_{j m}\left(t, t^{\prime}\right)
\end{aligned}
$$

are also independent of space.

Galilean invariance is the fundamental reason that the velocity correlators, hence the transport coefficients, are independent of space. The derivation given above is purely mathematical, relying on the basic freedom of choice of parameters $\left(\boldsymbol{R}, \boldsymbol{R}^{\prime}, \tau, \tau^{\prime}, \boldsymbol{\xi}\right)$, but we can also understand the results more physically. $\boldsymbol{X}$ and $\boldsymbol{X}^{\prime}$ can be thought of as the location of the origin of a comoving observer at times $t$ and $t^{\prime}$, respectively. GI implies that the velocity correlators measured by the comoving observer at her origin at times $t$ and $t^{\prime}$ must be equal to the velocity correlators measured by any comoving observer at her origin at times $t$ and $t^{\prime}$. In particular, this must be true for the observer in the lab frame, which explains equations (18), consequently equations (19). We can derive an expression for the GI mean EMF by using equations (19) for the transport coefficients in equation (6), and simplifying the integrands. Define

$$
\begin{aligned}
C_{j m l}\left(t, t^{\prime}\right) & =S_{j m l}\left(t, t^{\prime}\right)-2 A\left(t-t^{\prime}\right) \delta_{m 2} S_{j 1 l}\left(t, t^{\prime}\right) \\
D_{j m}\left(t, t^{\prime}\right) & =R_{j m}\left(t, t^{\prime}\right)+2 A t^{\prime} \delta_{m 2} R_{j 1}\left(t, t^{\prime}\right)
\end{aligned}
$$


Then the mean EMF, $\mathcal{E}(\boldsymbol{x}, t)$, can be written compactly as

$$
\begin{aligned}
\mathcal{E}_{i} & =\epsilon_{i j m} \int_{0}^{t} d t^{\prime} C_{j m l}\left(t, t^{\prime}\right) H_{l}^{\prime} \\
& -\int_{0}^{t} d t^{\prime}\left[\epsilon_{i j l}-2 A\left(t-t^{\prime}\right) \delta_{l 1} \epsilon_{i j 2}\right] D_{j m}\left(t, t^{\prime}\right) H_{l m}^{\prime}
\end{aligned}
$$

The mean field equation (1) for $\boldsymbol{H}(\boldsymbol{x}, t)$ is

$$
\frac{\partial H_{i}}{\partial t}+2 A \delta_{i 2} H_{1}=(\nabla \times \mathcal{E})_{i}+\eta \nabla^{2} H_{i}
$$

where $(\boldsymbol{\nabla})_{p} \equiv \partial / \partial X_{p}=\left(\partial / \partial x_{p}+2 A t \delta_{p 1} \partial / \partial x_{2}\right)$. We use equation (21) to evaluate $(\nabla \times \mathcal{E})_{i}$ :

$$
\begin{aligned}
(\nabla \times \mathcal{E})_{i}= & \int_{0}^{t} d t^{\prime}\left[C_{i m l}-C_{m i l}\right]\left[H_{l m}^{\prime}+2 A t \delta_{m 1} H_{l 2}^{\prime}\right] \\
+ & \int_{0}^{t} d t^{\prime} D_{j m}\left\{H_{i j m}^{\prime}+2 A t \delta_{j 1} H_{i 2 m}^{\prime}\right. \\
& \left.-2 A\left(t-t^{\prime}\right) \delta_{i 2}\left[H_{1 j m}^{\prime}+2 A t \delta_{j 1} H_{12 m}^{\prime}\right]\right\}
\end{aligned}
$$

Equations 22 and (23) form a closed set of integro-diffential equations governing the dynamics of the mean field, $\boldsymbol{H}(\boldsymbol{x}, t)$, valid for arbitrary values of $A$. The most visible properties of equation (23) for $(\nabla \times \mathcal{E})$ are: (i) Only the part of $C_{i m l}\left(t, t^{\prime}\right)$ that is antisymmetric in the indices $(i, m)$ contributes. Indeed both $S_{i m l}$ and $C_{i m l}$ can vanish for non helical velocity fluctuations, in which case dynamo action is determined only by the $D_{j m}$ terms. (ii) The $D_{j m}\left(t, t^{\prime}\right)$ terms are such that $(\boldsymbol{\nabla} \times \mathcal{E})_{i}$ involves only $H_{i}$ for $i=1$ and $i=3$, whereas $(\nabla \times \mathcal{E})_{2}$ depends on both $H_{2}$ and $H_{1}$. Together with the mean field induction equation 22 this means that the equations determining the time evolution of $H_{1}$ and $H_{3}$ are closed. Thus $H_{1}(\boldsymbol{x}, t)$ (or $H_{3}(\boldsymbol{x}, t)$ ) can be computed by using only the initial data $H_{1}(\boldsymbol{x}, 0)$ (or $H_{3}(\boldsymbol{x}, 0)$ ). The equation for $H_{2}$ involves both $H_{2}$ and $H_{1}$, and can then be solved.

The implications for the original field, $\boldsymbol{B}(\boldsymbol{X}, \tau)$, can be read off, because it is equal to $\boldsymbol{H}(\boldsymbol{x}, t)$ component-wise (i.e $\left.B_{i}(\boldsymbol{X}, \tau)=H_{i}(\boldsymbol{x}, t)\right)$. Thus, the $D_{j m}\left(t, t^{\prime}\right)$ terms do not couple either $B_{1}$ or $B_{3}$ with any other components, excepting themselves. In demonstrating this, we have not assumed that either the shear is small, or that $\boldsymbol{H}(\boldsymbol{x}, t)$ is such a slow function of time that it can be pulled out the time integrals in equations (21) and 23). Comparing with earlier work (where, essentially, both assumptions have been made) we conclude that there is no shear-current assisted dynamo of the form discussed by $[4,6,7]$, where there is explicit coupling of $B_{2}$ and $B_{1}$ in the evolution equation for $B_{1}$. Our calculations are based on a non perturbative treatment of shear, and this makes for a basic departure from earlier work which have treated shear perturbatively. Even when the shear is weak, two fluid elements which were close together initially would be separated by arbitrarily large distances at late times. Thus the two-time correlators, which appear naturally in the dynamo problem, have to be handled carefully in the presence of shear. Moreover, the perturbative treatment of shear is not guaranteed to preserve GI, which is a natural and fundamental ingredient of our non perturbative approach.

In conclusion we find that systematic use of the shearing coordinate transformation and the Galilean invariance of a linear shear flow allows us to develop a quasilinear theory of the shear dynamo which, we emphasize, is non perturbative in the shear parameter. Specifically, we have proved that there is essentially no shear-current assisted dynamo in the quasilinear limit when FOSA is applicable. Moreoever, our results are valid for any GI velocity statistics, independent of the forces (Coriolis, buoyancy etc) governing the dynamics of the velocity field. However, large-scale non helical dynamos (i.e. with no initially imposed kinetic helicity) are not ruled out, and further progress requires developing a dynamical theory of velocity correlators in shear flows.

We acknowledge Nordita for providing a stimulating atmosphere during the program on 'Turbulence and Dynamos'. We thank Axel Brandenburg, Karl-Heinz Rädler and Matthias Rheinhardt for valuable comments.

* Electronic address: ssridhar@ rri.res.in

$\dagger$ Electronic address: kandu@iucaa.ernet.in

[1] A. Brandenburg et al., Astrophys. J., 676, 740 (2008).

[2] T. A. Yousef et al., Phys. Rev. Lett., 100, 184501 (2008); Astron. Nachr. 329, 737 (2008).

[3] P. J. Käpylä, M. J. Korpi and A. Brandenburg, Astron. Astrophys., 491, 353 (2008); arXiv:0812.1792 (2008); D. W. Hughes and M. R. E. Proctor, arXiv:0810.1586 (2008).

[4] I. Rogachevskii and N. Kleeorin, Phys. Rev. E 68, 036301 (2003); 70046310 (2004); Astron. Nachr., 329, 732 (2008).

[5] A. A. Schekocihin et al., arXiv:0810.2225 (2008).

[6] K.H. Rädler and R. Stepanov, Phys. Rev E 73, 056311 (2006).

[7] G. Rüdiger and L. L. Kitchatinov, Astron. Nachr. 327, 298 (2006).

[8] H. K. Moffatt, Magnetic Field Generation in Electrically Conducting Fluids, Cambridge University Press, Cambridge (1978); F. Krause, K.-H. Rädler, Mean-field magnetohydrodynamics and dynamo theory, Pergamon Press, Oxford (1980); A. Brandenburg and K. Subramanian, Phys. Rep. 417, 1 (2005). 